\title{
Multilayer Matching of Metric Structures Using Hierarchically Well-separated Trees
}

\author{
Yusuf Osmanlıoğlu**, Ali Shokoufandeh \\ Department of Computer Science, Drexel University, 3141 Chestnut St., Philadelphia, PA, 19104, USA
}

\section{ABSTRACT}

\begin{abstract}
We consider the matching problem between two metric distributions where establishing a one-to-one matching of features may not always be possible. Although many-to-many graph matching techniques achieve the desired multi map between features, they ignore the spatial structure of the nodes. We propose a novel technique, multilayer matching, for solving the matching problem which utilizes both the individual node features and the clustering information of nodes. Our method uses the hierarchically well-separated trees (HSTs) to represent the metric distribution such that non-leaf nodes in the tree representation corresponds to a constellation of features in the original structure. By using HSTs in a linear programming setup, we obtain a matching between features through finding a mapping between non-leaf nodes among the two HSTs. We further provide a primal-dual approximation algorithm for the multilayer matching which runs several order of magnitudes faster while achieving comparable success rates. Application of the method to the image matching problem is also presented in the paper. Empirical evaluation of the method and its primal-dual extension on a set of recognition tests show the robustness and efficiency of the overall approach.
\end{abstract}

(C) 2016 Elsevier Ltd. All rights reserved.

\section{Introduction}

Matching of metric distributions is a fundamental problem in computer science which has numerous real life applications such as image matching and object tracking. Since arbitrary metrics can be represented using graph structures, the problem can be reduced to graph matching, i.e., given two graphs $G$ and $H$, find a mapping among vertices and edges of graphs. Exact graph matching is known to be computationally intractable. Specifically, subgraph isomorphism which asks whether $G$ contains an induced subgraph $H^{\prime}$ that is isomorphic to $H$, is a problem known to be NP-complete (Cook, 1971). Even a simpler special case of the problem, graph isomorphism, is neither known to be polynomial time solvable nor NP-complete. These facts led the research in the field to focus on inexact matching since early 80s (Shapiro and Haralick, 1981).

Aside from inexact matching being a feasible way to attack the intractable problem of exact graph matching, it might even be desirable in some problem domains where a node in one graph should be matching to more than one node in the other graph. Image matching is an instance of this where image features are represented as graph nodes and relationships among features are structured with weighted edges. In this setup, fea-

\footnotetext{
** Corresponding author:

e-mail: osmanlioglu@cs.drexel.edu (Yusuf Osmanlığlu)
}

tures of two images representing the same object can have minor differences due to occlusion, noise, scaling, etc. As a result, establishing a mapping between sets of nodes amongst two graphs rather than establishing a one-to-one matching between individual nodes becomes necessary.

Metric embedding based methods are among the most popular approaches for tackling many-to-many matching problem (Keselman et al., 2003). Here, the objective is mapping data from a source space to a "simpler" target space while preserving the distances. Using embedding, a graph that is equipped with a metric can be approximated as a tree structure. It is well known that approximating the solution for many NPhard problems in general metrics can be done in polynomial time once data is embedded into tree metrics. However, such embeddings tend to introduce distortion. A common technique for overcoming high distortion rates is probabilistic approximation method of Karp (Karp, 1989). Utilizing probabilistic embedding, Bartal introduced the notion of hierarchically wellseparated trees (HST) where edge weights on a path from root to leaves decrease by a constant factor in successive levels (Bartal, 1996). Embedding into HSTs improves the representational power of graphs especially in domains such as image matching since the internal nodes of the tree represents constellations of nodes of the original graph. Capturing the segmentation information at internal nodes of the tree along with the node features at its leaves make HSTs useful tools for inexact matching. 
It is an underlying assumption that many-to-many matching algorithms use the features associated with individual nodes to establish the correspondences, while ignoring the underlying regions containing the spatial distribution of the matched features. In earlier work, we proposed a novel matching method, multilayer matching, that uses spatial distribution of nodes in addition to individual features to tackle the matching problem (Osmanlıoğlu and Shokoufandeh, 2015). Motivated by the approach of Kleinberg and Tardos $(\mathrm{K} \& \mathrm{~T})$ for solving the metric labeling problem, our method uses optimization over HSTs (Kleinberg and Tardos, 2002). We first embed two given graphs into separate HSTs and then match the nodes of the resulting trees at fixed layers by solving a linear program (LP) that is similar to the formulation of K\&T. Obtaining a matching between the internal nodes of the HSTs makes it possible to take clustering structure of the features into account in addition to the individual image features. Utilizing the spatial distribution of features improves the running time of the matching task while accuracy is achieved by the use of feature vectors throughout the process.

Our method requiring to solve an LP makes the running time to be bound to number of variables and constraints of the LP formulation, and the efficiency of the LP solver. In this paper, we improve the performance of the method by presenting a primal-dual approximation algorithm for the multilayer matching problem (Goemans and Williamson, 1997). Our primaldual algorithm achieves a speed up by of a factor up to $500 \mathrm{x}$ while obtaining comparable matching scores relative to baseline algorithms and other graph based methods from the pattern recognition literature. We demonstrate the utility of the method in the context of image matching. Experimental results indicate that the proposed algorithms perform well for standard datasets.

The rest of the document is organized as follows: Section $\$ 2$ gives an overview of notations and definitions. In Section $\S 3$, we present the details of multilayer matching which is followed by a primal-dual approximation algorithm for the problem in Section $\S 4$. Next, we present the application of both methods to image matching problem in Section $§ 5$. Finally, Section $§ 6$ concludes the document.

\section{Background and Definitions}

Over the last three decades, several methods were proposed for tackling the inexact graph matching problem including tabu search (Williams et al., 1999), error-correcting graph matching (Bunke, 1999), and convex optimization formulations (Almohamad and Duffuaa, 1993). Graph edit distance based methods are among widely studied approaches for addressing noisy, many-to-many matching (Ambauen et al., 2003; Berretti et al., 2004). This approach performs local searches over the graph and transforms (part of) one graph into the other by minimal cost modifications such as inserting, deleting, substituting, or merging of nodes and edges. Using spectral graph theory, Caelli and Kosinov approached the problem as eigen-decomposition of adjacency matrix representation of the graph (Caelli and Kosinov, 2004). Zaslavskiy et al. formulated many-to-many graph matching as a discrete optimization problem and pro- posed an approximation algorithm based on continuous relaxation (Zaslavskiy et al., 2010). Another technique for dealing with the problem is by incorporating metric embedding methods. Although used in a different problem domain, pyramidal graph representation of Haxhimusa et al. can be contrasted to our multilayer matching approach (Haxhimusa et al., 2009).

Our approach to the inexact graph matching brings three major tools together from the domains of probabilistic embedding, operations research, and approximation algorithms. We present a summary of the tools in the rest of this section.

\subsection{Metric Embedding of Graphs into HSTs}

The term embedding refers to a mapping between two spaces (Indyk, 2001). Given a hard computational problem, the objective of embedding methods is to reduce the problem into another space where a tracktable (approximate) solution is possible. Thus, embedding techniques are commonly used for finding approximate solutions to NP-hard problems. A finite metric space ${ }^{1}(P, d)$ can naturally be represented as a weighted graph $G=(V, E)$ with shortest path as the distance measure where points in $P$ form the vertex set $V$ and pairwise distances among points become the edge weights. Embedding of graphs into simpler structures is of particular interest since many problems involving graphs are known to be intractable. Simplifying graphs mainly involve operations such as removing edges that change the distance metric of the graph, removing or adding vertices, or changing weights of edges. This approach, however, introduces distortion on the distances defined over the graph.

In general, if not possible it is hard to find an isometric embedding between two arbitrary metric spaces. Therefore, it is important to find an embedding in which the distances between vertices of the destination metric are as close as possible to their counterparts in the source metric space. Trees are a feasible target space for many problems involving graphs since solving problems over trees is relatively easier compared to arbitrary graphs. Embedding of graphs into trees is a very challenging problem; even for the simple case of embedding an $n$-cycle into a tree. Karp introduced the notion of probabilistic embedding for overcoming this difficulty. Given a metric $d$ defined over a finite space $P$, the main idea is to find a set $S$ of simpler metrics defined over $P$ which dominates $d$ and guarantees the expected distortion of any edge to be small (Karp, 1989).

Uniform metrics are among the simplest tessellation spaces where all distances are regularly distributed across cells. Problems defined under such metrics can be easily solved by applying divide-and-conquer approaches which makes uniform metrics important from a computational point of view. Motivated by these observations, Bartal defined the notion of hierarchically well separated trees (HST) for viewing finite metric spaces as a uniform metric (Bartal, 1996). A $k$-HST is defined as a rooted weighted tree where edge weights from a node to each

\footnotetext{
${ }^{1}$ Given a set of points $P$, a mapping $d: P \times P \rightarrow R^{+}$is called distance function if $\forall p, q, r \in P$ the following four conditions are satisfied: $d(p, q)=0$ iff $p=q, d(p, q) \geq 0, d(p, q)=d(q, p)$, and $d(p, q)+d(q, r) \geq d(p, r)$. The pair $(P, d)$ is called a metric space or a metric.
} 
of its children are the same and decrease by a factor of at least $k$ along any root to leaf path. Assuming that the maximum distance between any pair of points (diameter) in source space is $\Delta$, source space is separated into clusters (sub-metrics) of diameter $\frac{\Delta}{k}$. The resulting clusters are then linked to the root as child nodes with edges of weight $\frac{\Delta}{2 k}$. The relation between parent and child nodes continues recursively until the children nodes consist of single data elements. Bartal has shown the lower bound for distortion of embedding into HSTs to be $\Omega(\log n)$ and Fakcharoenphol et al. (Fakcharoenphol et al., 2003) introduced a deterministic embedding algorithm that achieves a tight distortion rate $(\Theta(\log n))$.

\subsection{Metric Labeling Problem}

One of the earliest algorithmic application of HST in general family of classification problems is known as metric labeling (Kleinberg and Tardos, 2002). Given a set of objects $O$ and a set of labels $\mathcal{L}$ with pairwise relationships defined among the elements of both sets, the goal is to assign a label to each object by minimizing a cost function involving both separation and assignment costs. Separation cost penalizes assigning loosely related labels to closely related objects while assignment cost penalizes labeling an object with an unrelated label. Although, there has been ample studies on solving classification problems using labeling methods, their work was the first that provided a polynomial-time approximation algorithm with a nontrivial performance guarantee.

Metric labeling can be formulated as a quadratic optimization problem where the contribution of the separation cost would involve quadratic terms. Kleinberg and Tardos used embedding of label graph into an HST and measured the distances between labels over the tree which made it possible to linearize the objective function (Kleinberg and Tardos, 2002). They formulated the problem as a linear program (LP) and solving it, obtained a fractional mapping. Following a supplementary deterministic rounding procedure, a many-to-one matching from object set to label set is achieved. Distortion rate of K\&T's method is bound to the distortion of HST embedding since HSTs are being used both in the solution to the LP and in rounding of the fractional solution. Utilizing the HST embedding algorithm of (Fakcharoenphol et al., 2003) in K\&T's method, one can achieve $O(\log n)$ overall distortion rate. Recently, we extended the original metric labeling to a generalized formulation in the domain of user association in celular networks (Wildman et al., $2015 a, b)$. The goal is to assign users to basestations which offer best downlink rate while optimizing congestion by keeping the user distribution over basestations balanced.

\subsection{Primal-Dual Algorithms}

Over a half century, linear programming has been used for solving combinatorial optimization problems albeit with approximate results. Most of the combinatorial optimization problems can be modeled with integer linear programming (ILP) formulations. Since ILP is known to be NP-hard to solve, such problems are approximated by first relaxing the integrality conditions of integer program and then solving the resulting linear program. As an auxiliary step, fractional solution of the linear program is post-processed with a rounding algorithm to obtain discrete results. Thus, it is necessary to find a suitable rounding algorithm with a bounded distortion guarantee.

Since the early days of linear programming, primal-dual has been used as one of the polynomial time methods for solving linear programs (Dantzig et al., 1956). From the combinatorial optimization perspective, the main asset of this method is that it allows a weighted optimization problem to be reduced to a purely combinatorial problem. A modified version of standard primal-dual method was proposed by Goemans and Williamson for finding approximate solutions to various network design problems without the need for a supplementary rounding phase (Goemans and Williamson, 1997). The modified primal-dual algorithm differs from the original form in that complementary slackness conditions are relaxed. The algorithm attains an approximation to the original problem and a feasible solution to its dual in the meantime. The main advantage of using modified primal-dual method over classical linear programming relaxation followed by rounding is twofold. First, it runs faster while guaranteeing a bounded distortion rate. Second, if the data changes during the course of algorithm running, the algorithm can recover by updating only the newly violated constraints without the need for solving the problem from scratch.

\section{Multilayer Matching}

We now present a novel technique for solving the multilayer matching, which we will refer to as MM in the rest of the text. Our objective is to obtain a correspondence between metric distributions by using spatial structure of data in addition to individual node features. Our technique extends the metric labeling formulation of K\&T.

Given two metric distributions represented as an object graph $G_{O}$ and a label graph $G_{\mathcal{L}}$, the goal is to match clusters of $G_{O}$ to those of $G_{\mathcal{L}}$ as demonstrated in the first row of Fig. 1. We begin by embedding both graphs into separate HSTs $\mathcal{T}_{O}$ and $\mathcal{T}_{\mathcal{L}}$ using the deterministic embedding algorithm presented in (Fakcharoenphol et al., 2003). The embedding expands the tree until the leaf nodes of the trees correspond to individual nodes in the original graphs. Specifically, given that the diameter of the graph is $\Delta$, the resulting $k$-HST will have at most $\left\lceil\log _{k} \Delta\right\rceil$ layers. Note that the internal nodes of the HSTs introduced by the embedding, correspond to subsets (clusters) of nodes in the original graph. Then, by finding correspondences over the nodes of HSTs at a fixed layer as shown in second row of Fig. 1, we obtain a mapping between clusters of nodes in the original graphs. For example, if we were to match the second layer of the left-tree with the third layer of the right-tree in Fig. 1, we would expect non-leaf nodes $a 2$ and $a 3$ to get a mapped to $b 8$ and $b 9$, respectively. Referring to the set of (nonleaf) nodes at a fixed layer $i$ of tree $\mathcal{T}$ as $\mathcal{T}^{i}$, our goal can be restated as matching $\mathcal{T}_{O}^{i}$ with $\mathcal{T}_{\mathcal{L}}^{j}$. Thus, leaves that are descendants of matched non-leaf nodes will be assigned to each other in a many-to-many fashion. 


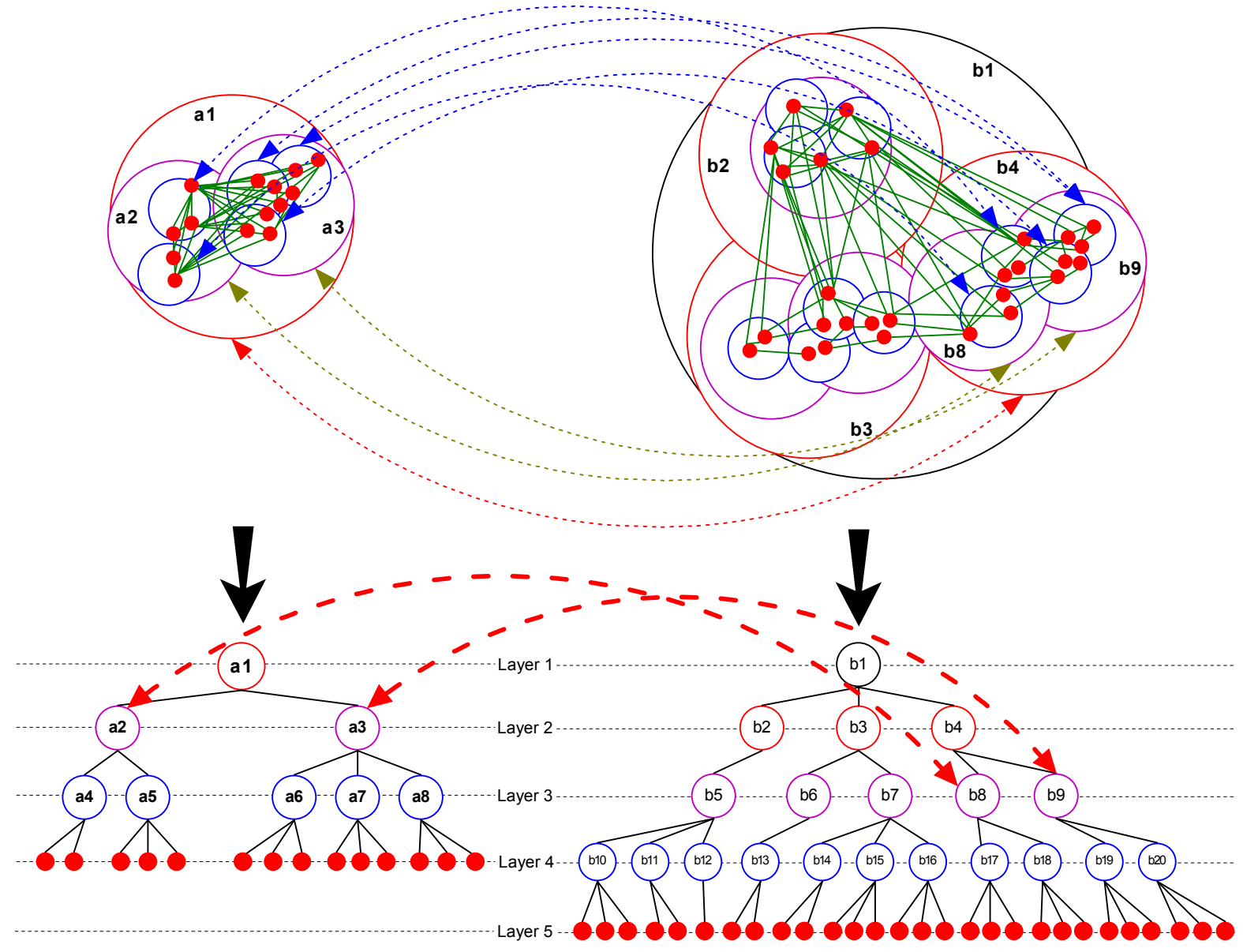

Fig. 1. Given the two graphs in the first row, our method first embeds them into separate HSTs using Fakcharoenphol et al.'s algorithm, resulting with the $k$-HSTs shown in the second row (Fakcharoenphol et al., 2003). Then we match non-leaf nodes of both trees using an extended version of metric labeling.

The quadratic optimization problem (IQP) for the MM with integrality constraint, denoted $M M_{I Q P}$, can be stated as follows:

$$
\begin{array}{ll}
\min & \sum_{p \in \mathcal{T}_{o}^{i}} \sum_{a \in \mathcal{T}_{\mathcal{L}}^{j}} c_{p, a} \cdot x_{p, a}+\sum_{p, q \in \mathcal{T}_{o}^{i}} \sum_{a, b \in \mathcal{T}_{\mathcal{L}}^{j}} w_{p, q} \cdot d_{a, b} \cdot x_{p, a} \cdot x_{q, b} \\
\text { s.t. } & \sum_{a \in \mathcal{T}_{\mathcal{L}}^{j}} x_{p, a}=1, \quad \forall p \in \mathcal{T}_{O}^{i} \\
& x_{p, a} \in\{0,1\}, \quad p \in \mathcal{T}_{O}^{i}, a \in \mathcal{T}_{\mathcal{L}}^{j}
\end{array}
$$

where $i$ and $j$ are the layers to be matched at each tree, $c_{p, a}$ is the cost and $x_{p, a}$ is the probability of assigning object node $p \in \mathcal{T}_{O}^{i}$ to label node $a \in \mathcal{T}_{\mathcal{L}}^{j}, w_{p, q}$ is the measure of correlation between object nodes $p, q$ in $\mathcal{T}_{O}$, and $d_{a, b}$ is the distance between label nodes $a$ and $b$ over the HST $\mathcal{T}_{\mathcal{L}}$. One can solve the $M M_{I Q P}$ by relaxing the integrality condition over $x_{p, a}$ variables. Discrete label assignments can be obtained by applying a rounding procedure to the fractional assignments of the relaxed IQP.

Motivated by the linearization of metric labeling formulation by $\mathrm{K} \& \mathrm{~T}$, we state the following integer linear program- ming (ILP) formulation for MM, denoted $M M_{I L P}$ :

$$
\begin{array}{lr}
\min \sum_{p \in \mathcal{T}_{o}^{i}} \sum_{a \in \mathcal{T}_{\mathcal{L}}^{j}} c_{p, a} \cdot x_{p, a}+\sum_{p, q \in \mathcal{T}_{O}^{i}} w_{p, q} \cdot D_{p, q} & \\
\text { s.t. } \sum_{a \in \mathcal{T}_{\mathcal{L}}^{j}} x_{p, a}=1, & \forall a \in \mathcal{T}_{O}^{i} \\
& D_{p, q}=\sum_{T \in \mathcal{T}_{\mathcal{L}}^{1 \cdots j}} l_{T} \cdot \bar{x}_{p, q, T}, \\
\bar{x}_{p, q, T} \geq \tilde{x}_{p, T}-\tilde{x}_{q, T} \\
\bar{x}_{p, q, T} \geq \tilde{x}_{q, T}-\tilde{x}_{p, T} \\
x_{p, a} \in\{0,1\}, \quad \forall p, q \in \mathcal{T}_{O}^{i}
\end{array}
$$

where $l_{T}$ is the length of the edge between node $T$ and its parent in an HST, and $\tilde{x}_{p, T}$ is the joint probability of objects in set $p$ getting assigned to the labels included in the subtree rooted at $T$. Note that, if the HST $\mathcal{T}_{O}$ was expanded in $n$ layers, $p \in \mathcal{T}_{O}^{i}$ refers to an internal node of the tree $\mathcal{T}_{O}$ for $i<n$ and to a leaf node for $i=n$ which corresponds to a cluster of nodes and an individual node in the original graph $G_{O}$, respectively. 
Calculating the assignment $\operatorname{cost} c_{p, a}$ between sets $p \in \mathcal{T}_{O}^{i}$ and $a \in \mathcal{T}_{\mathcal{L}}^{j}$ to match two clusters requires a measure of set similarity. In our experiments, we used normalized Hausdorff distance 2 since it has been successfully applied in the image matching domain (Huttenlocher et al., 1993). The correlation value $w_{p, q}$ between object nodes $p, q \in \mathcal{T}_{O}$ is set to be the reciprocal of distance between the two nodes over $\mathcal{T}_{O}$.

Proposition 1. $M M_{I Q P}$ and $M M_{I L P}$ are equivalent.

Proof. We first consider the objective functions. Note that the contribution of the assignment cost is the same for both problems. In the separation cost of the $M M_{I Q P}, w_{p, q}$ is multiplied by $\sum_{a, b \in \mathcal{T}^{j}} d_{a, b} \cdot x_{p, a} \cdot x_{q, b}$ whereas the same term is multiplied by $\sum_{T} l_{T} \cdot\left|\tilde{x}_{p, T}-\tilde{x}_{q, T}\right|$ in $M M_{I L P}$. Observe that, for $x_{p, a} \in\{0,1\}$, the latter expression gives the distance between labels $a, b \in \mathcal{T}_{\mathcal{L}}$ that $p$ and $q$ are assigned to. Thus, the separation cost of the two formulations are also equivalent. Next, we compare the constraints. $M M_{I Q P}$ only have type $x_{p, a}$ variables. The only free variables in $M M_{I L P}$ are of type $x_{p, a}$ since the variables of type $\bar{x}_{p q T}$ and $\tilde{x}_{q, T}$ take values depending on corresponding $x_{p, a}$ variables. Constraint (2a) of $M M_{I L P}$ is identical to that of $M M_{I Q P}$. The constraints of type ( $2 \mathrm{~b})$ in $M M_{I L P}$ calculate the distance between the assigned labels of the two object nodes by utilizing the type (2c) of constraints which simulate the absolute value function in linear programming terms. Since both formulations restrict the only independent variables $x_{p, a}$ to take binary values, the two programs are equivalent.

We relax the integrality condition of the ILP formulation which yields the LP formulation $\left(M M_{L P}\right)$. Solving $M M_{L P}$ in polynomial time, we will obtain a fractional solution to the problem where each node of $\mathcal{T}_{O}^{i}$ gets mapped to at least one node of $\mathcal{T}_{\mathcal{L}}^{j}$. One can obtain discrete assignments between object and label nodes by applying the rounding procedure presented by K\&T.

\section{Primal-Dual Algorithm}

Solving $M M_{L P}$ is impractical for problems which involve distributions with large number of features. Since problems such as image matching can easily involve more than hundreds of features for a single image, a faster algorithm for multilayer matching is desirable. In this section, we present a primal-dual approximation algorithm to improve running time performance.

\footnotetext{
${ }^{2}$ Given a metric space $(M, d)$ and two subsets $X, Y \in M$, the symmetric Hausdorff distance $d_{H}(X, Y)$ between $X$ and $Y$ is defined as $d_{H}(X, Y)=$ $\max \left\{\max _{x \in X} \min _{y \in Y} d(x, y), \max _{y \in Y} \min _{x \in X} d(x, y)\right\}$.
}

We first state the dual of the $M M_{L P}$ as follows:

$$
\begin{aligned}
& \max \sum_{p \in \mathcal{T}_{O}^{i}} y_{p} \\
& \text { s.t. } \quad y_{p}+\sum_{\substack{q \in \mathcal{T}_{O}^{i} \\
T: a \in T}} \bar{y}_{p, q, T}-\sum_{\substack{q \in \mathcal{T}_{O}^{i} \\
T: a \in T}} \bar{y}_{q, p, T} \leq c(p, a), \\
& \forall p \in \mathcal{T}_{O}^{i}, a \in \mathcal{T}_{\mathcal{L}}^{j} \\
& -\bar{y}_{p, q, a}-\bar{y}_{q, p, a}+l_{a} \cdot \tilde{y}_{p, q} \leq 0, \forall p, q \in \mathcal{T}_{O}^{i}, a \in \mathcal{T}_{\mathcal{L}}^{j} \\
& -\tilde{y}_{p, q} \leq w_{p, q}, \quad \forall p, q \in \mathcal{T}_{O}^{i} \\
& y_{p}, \tilde{y}_{p, q} \text { unrestricted, } \\
& p, q \in \mathcal{T}_{O}^{i} \\
& \bar{y}_{p, q, a} \geq 0 \text {, } \\
& p, q \in \mathcal{T}_{O}^{i}, a \in \mathcal{T}_{\mathcal{L}}^{j}
\end{aligned}
$$

where constraints of type (3a),(3b), and (3c) correspond to the primal variables of type $x_{p a}, \bar{x}_{p q T}$, and $D_{p q}$, respectively. Note that the LP formulation we analyze here differs from the problems investigated by the primal-dual method of G\&W in several ways. First, in contrast to constraints being homogeneous in the problems of G\&W method, our LP formulation has three interdependent types of constraints. Thus, the order in which we identify, iterate between, and modify violated primal constraints, their corresponding dual variables, and dependent dual variables should be acyclic. Second, contrary to non-negativity of dual variables in the G\&W method, our formulation has unrestricted dual variables which may require increasing or decreasing values depending on the type of the primal constraint violation. Third, the G\&W method attains a dual feasible solution by initializing dual variables to 0 while maintaining slackness in all dual constraints. Due to constraints of type (3c), we require initializing some of the dual variables with negative values to maintain dual constraint slackness at the start of our algorithm. Finally, the G\&W method enforces dual feasibility throughout the algorithm whereas we must relax this condition for the constraints of type (3a) which previously have become tight.

Our primal-dual algorithm is presented in Alg. 1. It starts by initializing primal and dual variables, assignment cost function $c(\cdot, \cdot)$ as the Hausdorff distance between nodes, and pairwise relation function $w$ as the reciprocal of the distance between object nodes on the HST $\mathcal{T}_{O}$ (line 1). The algorithm also initializes the set $\mathcal{P}$ of violated primal constraints of type (2a) in line 2 . Initially, the only violated primal constraints will be of type (2a) and the algorithm will terminate once these violations are fixed. Each iteration of the while loop resolves one such violation without introducing any additional violations of the same type (lines $3-9$ ). Fixing of a violation is achieved by raising the value of dual variables $y_{p}$ corresponding to constraints of type (2a) until one of the constraints in (3a) becomes tight (line $4)$. The primal variable $x_{p a}$ corresponding to the newly tightened constraint in (3a) is set to 1 (line 5); this fixes a violated constraint in $\mathcal{P}$ (line 6). However, fixing one violation from set $\mathcal{P}$ introduces new primal constraint violations of type (2b) and (2c) that need to get fixed before proceeding to the next iteration. The order in which we fix the newly violated primal constraints is important in order not to violate an already fixed primal constraint of type (2a). First, assuming that $x_{p a}=1$ from 


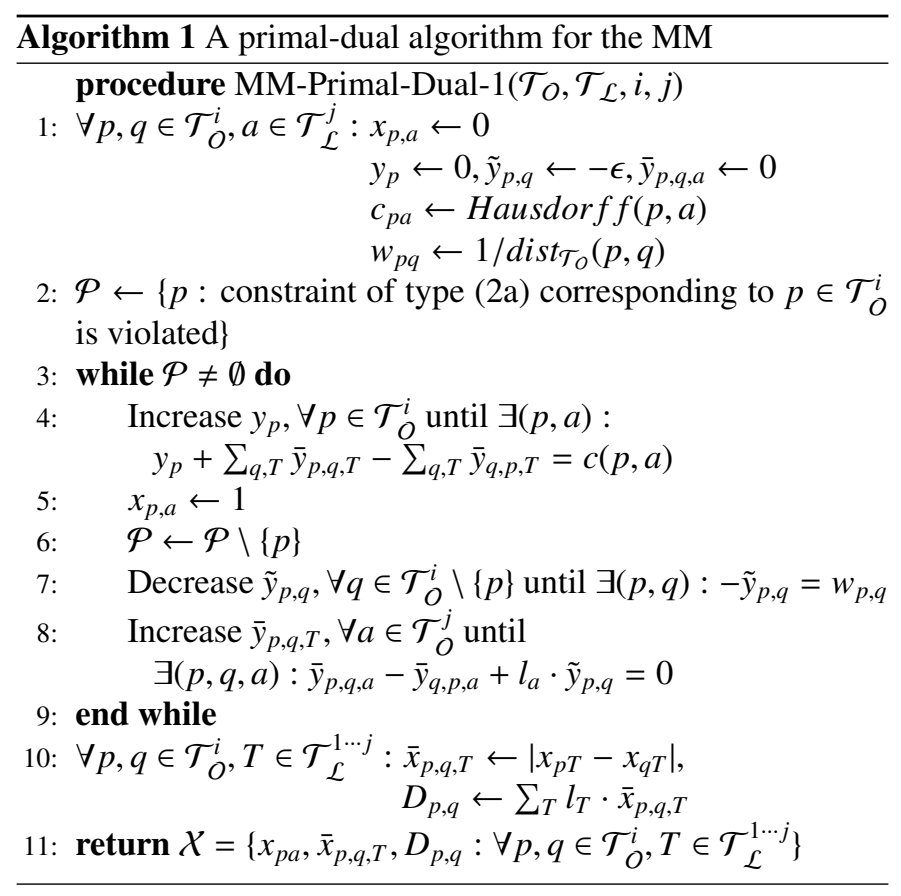

line 5, the dual variables of type $\tilde{y}_{p, q}$ that depend on $x_{p a}$ are decreased (line 7) until one of the constraints of type (3c) become tight. Then, variables of type $\bar{y}_{p, q, a}$ are increased until the corresponding constraint in (3b) becomes tight (line 8). Since the while loop primarily aims to resolve violated constraints of type (2a), other types of primal constraints may stay violated until the end of the algorithm although we raise the corresponding dual variables several times throughout the iteration of the while loop. Once the iteration of the loop finishes, feasible primal variables $\bar{x}_{p, q, a}$ and $D_{p, q}$ are constructed by using the values of integral primal variables $x_{p a}$ (line 10), yielding a feasible, integer solution to the primal problem $M M_{L P}$.

The algorithm can be implemented by representing the constraints by a matrix and then solving the inequalities row by row. However, such an implementation will be inefficient due to the constraint matrix being sparse. Consequently, we present an efficient primal-dual algorithm, denoted $M M_{P D}$, in Alg. 2. The algorithm starts with initializing assignment variables $x_{p a}$, set of unlabeled objects $\mathcal{P}$, and the cost and pairwise relation functions $c$ and $w$ as in the first algorithm. It further defines function $d$ which measures the distance between label nodes over the HST $\mathcal{T}_{\mathcal{L}}$ and an adjusted assignment cost function $\phi$ which comprises the backbone of the algorithm. The value of $\phi(p, a)$ is initially set to be the assignment cost of $p$ to $a$ (line 1). At each iteration of the loop in lines $2-7$, the algorithm makes an assignment for the object-label pair $(p, a)$ that minimizes the adjusted assignment cost function $\phi$ (lines $3-4$ ). Before proceeding to the next iteration, $\phi$ function is updated for each of the objects that are still unassigned by an amount of separation cost with respect to the recently assigned object. Algorithm iterates until no unassigned object node is left.

Proposition 2. Alg. 1 and Alg. 2 are equivalent.

Proof. Observe that in both algorithms, each iteration of the main loop assigns an object node to a label. The first algo-

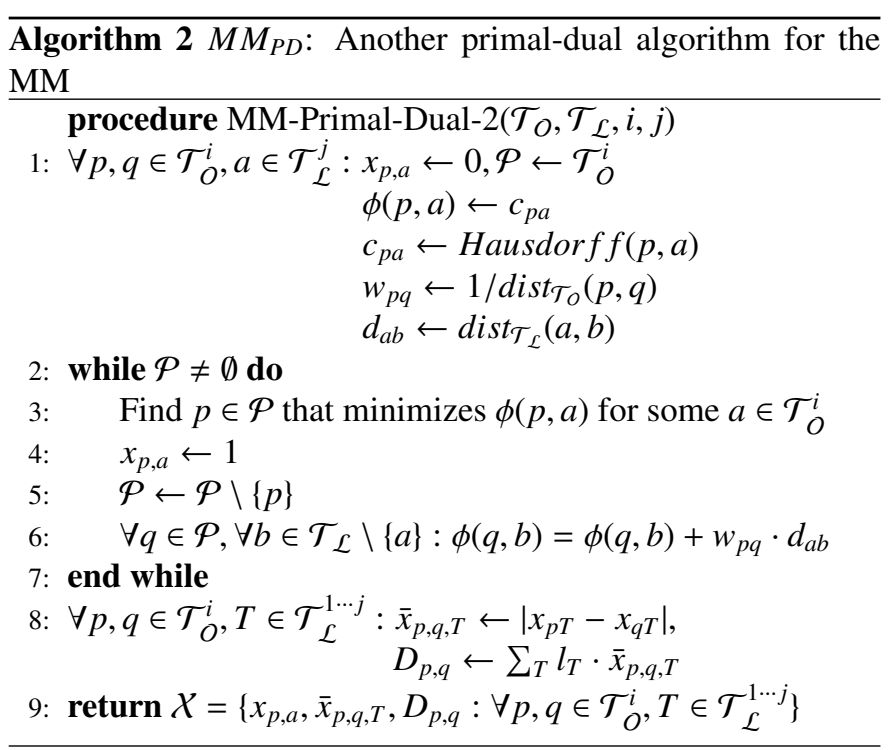

rithm iterates through violated constraints of type (2a), satisfying each by assigning an object node to a label node. The second algorithm achieves the same by directly looping over unassigned objects. Alg. 1 chooses the dual constraint that becomes tight as a result of increasing the dual variables of type $y_{p}$ (line 4) and makes the assignment for the corresponding primal variable (line 5). In Alg. 2, this is attained by choosing an unassigned object $p$ that minimizes $\phi_{p a}$ (line 3 ). These steps are equivalent in that one maintains the adjusted assignment costs by updating dual variables $\tilde{y}_{p q}$ and $\bar{y}_{p q a}$ (lines 7-8) whereas the other maintains them through the potential function $\phi$.

Proposition 3. Given that $\left|\mathcal{T}_{O}^{i}\right|=n$ and $\left|\mathcal{T}_{\mathcal{L}}^{j}\right|=m$, running time complexity of Alg. 2 is $O\left(n^{2} m+m^{2}\right)$.

Proof. Initialization phase takes $O\left(n^{2}+m^{2}+n m\right)$ time. Each iteration of the loop resolves one violation of constraint type (2a) and once a violation gets fixed, it will never get violated again for the rest of the execution. Thus, the loop is executed $n$ times, and lines 2,4, and 5 each take $O(n)$ time. Making an aggregate analysis for lines 3 and 6 , we get $O\left(n^{2}\right)$ and $O\left(n^{2} m\right)$ time, respectively. Thus, overall running time of the algorithm is $O\left(n^{2}+m^{2}+n m+n^{2} m\right)$ which is asymptotically equal to $O\left(n^{2} m+m^{2}\right)$.

Remark 1. Alg. 2 is a greedy algorithm in that at each iteration, the ( $p, a)$ pair that minimizes the adjusted assignment cost $\phi(p, a)$ is selected for assignment.

Remark 2. Observe that in Alg. 2, $\phi(p, a)$ is not updated for an object node $p$ once it gets assigned and that at the end of the algorithm, summation $\sum_{p a} \phi(p, a) x_{p a}$ gives the value of the objective function in (3).

\section{Application to Image Matching}

We apply the multilayer matching formulation of $\S 3$ to image matching problem. Given an image, our method starts with extracting SIFT features of the image (Lowe, 2004). Then, we 

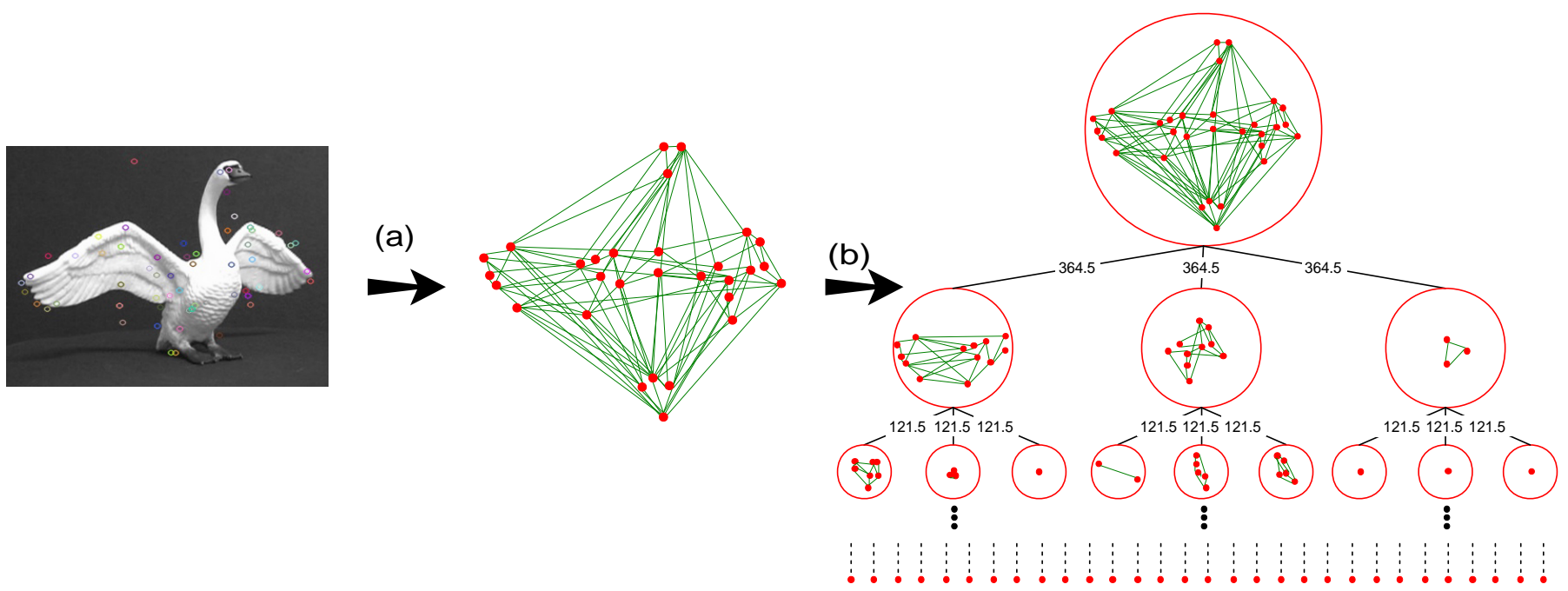

Fig. 2. (a) Graph representation of image features (b) Embedding the graph representation into 3-HST

represent the image as a complete undirected graph where features are represented by nodes and the relation between features are represented by edges. Next, we embed this complete graph into an HSTs as shown in Fig. 2. Having two such HSTs, the objective is to find a mapping between the internal nodes of the HSTs which represent a constellation of features of the images. Comparing layers that are closer to leaf layer yields a finer level of matching whereas matching layers closer to root runs faster albeit resulting in a coarse matching. This tradeoff between robustness and running time can be optimized by progressively altering the layers to match. Assume that given a dataset of images and a query object, we are asked to find the image that resembles the query object most. Using layered tree matching, we first match the query object with the rest of the images at a higher layer. This step is performed in short time since the trees consist of few nodes at this layer. Then, we discard the objects with large matching score and carry on matching at consecutive levels of the HSTs with the remaining images. Thus, progressively we eliminate the images that do not resemble the query image by rough HST matchings at coarser layers and perform rigorous matches with the few remaining images. We will refer to this method as sieve approach in the rest of this chapter.

\subsection{Experiments}

To demonstrate our approach on image matching problem, we performed experiments on COIL-20 (Nene et al., 1996) and MPEG-7 CE-Shape-1 (Part B) datasets. COIL-20 dataset consists of 1440 gray scale images of 20 objects where each object has 72 views obtained by consecutive rotations of 5 degrees around $z$ axis Fig. 3. MPEG-7 is a shape dataset containing silhouettes of 70 objects with 20 samples for each, having a total of 1400 shapes in the entire dataset. The experiments are carried using a leave-one-out procedure: a query image is removed from the test set and pairwise similarity scores between this image and the rest are recorded in a similarity matrix. Matching is considered successful if the image that is deemed to be most similar is a neighboring view of the query object for the COIL20 dataset and of the same object type for the MPEG-7 dataset.

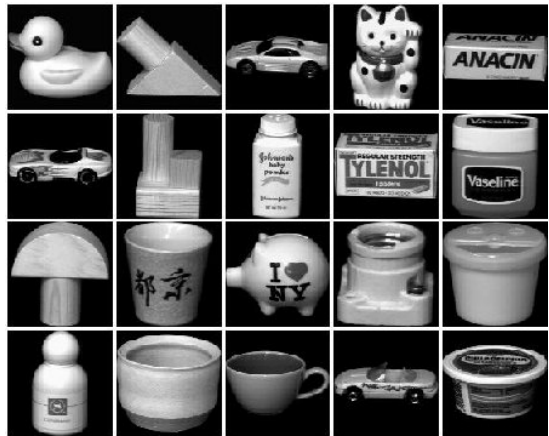

Fig. 3. Sample images from COIL-20 dataset (Nene et al., 1996).

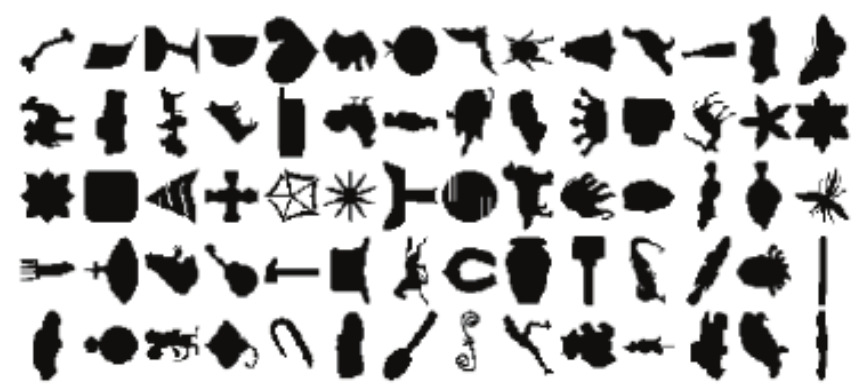

Fig. 4. Sample images from MPEG-7 CE-Shape-1 (Part B) dataset.

Experiments on COIL-20 dataset are carried out with four algorithms: $M M_{L P}, M M_{P D}$, and quadratic and linear programming formulations of the metric labeling problem (denoted $M L_{Q P}$ and $\left.M L_{L P}\right) . M M_{L P}$ and $M M_{P D}$ is run for fixed layers (1 through 5) for the entire dataset. We ran both algorithms a second time by applying the sieve approach over consecutive layers. We started running the sieve algorithm from the third layer since the accuracy of the matching is relatively low for the first two layers. While passing from third layer to the fourth, all but the most similar 100 images are discarded. Further 70 images are discarded during the transition from layer 4 to 5. $M L_{Q P}$ and $M L_{L P}$ are run to be used as the baseline. 


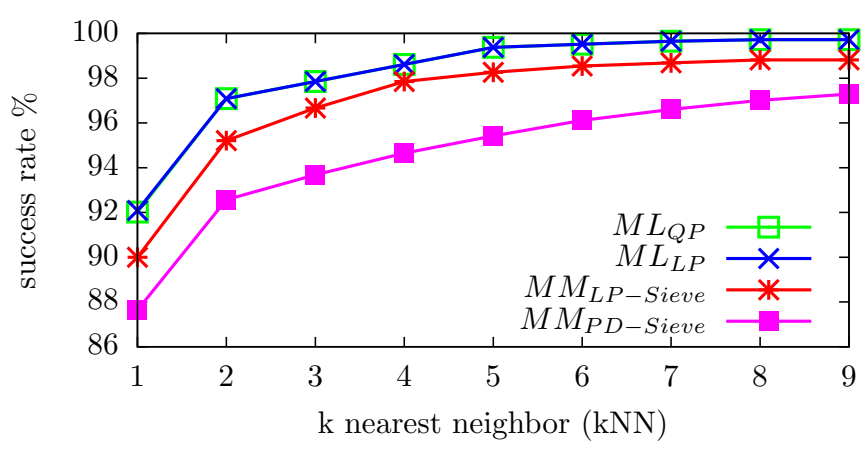

Fig. 5. Success rates for increasing number of neighboring views on COIL20 dataset experiment.

Results of the first experiment are presented in Fig. 5 and Tab. 1. Success rate for $M L_{Q P}$ and $M L_{L P}$ is identical except the $0.07 \%$ difference for $1-\mathrm{NN}$ case, whereas the former is 8 times faster than the latter. $M L_{L P}$ being as good as $M L_{Q P}$ is noteworthy since it shows that the $O(\log n)$ distortion which is introduced by HST embedding in LP formulation does not effect the result of matching for this dataset. As shown in the first column of Tab. 1, success rate of layered matching increases as the matching is carried out at finer layers. Ultimately, layered matching achieves $91.11 \%$ success for matching at the fifth layer which can be contrasted to the baseline success rate $92.08 \%$ for $\mathrm{kNN}=1$. Average running time of layered matching at fifth layer is almost three times more than the QP formulation although success rate is lower. On the other hand, applying the sieve to $M M_{L P}$ achieves a slightly lower success rate while reaching an average running time which is 3 and 25 times faster than baseline algorithms. Although success rate of $M M_{P D}$ is lower by $3.5 \%$ compared to $M M_{L P}$, its advantage lies in its running time performance which is over 20,65 , and 500 times faster than $M M_{L P}, M L_{Q P}$, and $M L_{L P}$, respectively. Speedup in our methods is an outcome of the multilayer approach which utilizes the spatial distribution of features and the primal dual approximation scheme which replaces the LP solver that is used in the baseline algorithm. Note that query images are identified with a different pose of the same object for $99 \%$ and $99.6 \%$ for $M M_{P D}$ and $M M_{L P}$.

Experiment on the MPEG-7 dataset is performed using the $M M_{P D}$ where we achieve an object recognition rate of $93.5 \%$ over the entire dataset. This result can be contrasted to graph matching based methods described in (Demirci et al., 2006) and (Demirci et al., 2011), which have success rates of $89.6 \%$ and $93.8 \%$, respectively. Note that, both methods proposed utilize the individual features in matching. Although the latter method outperformed $M M_{P D}$ by $0.3 \%$, our method achieves significant performance gain through using spatial distribution of features in addition to individual feature vectors. Specifically, both methods of Demirci et al. has high computational complexity due to employing the earth mover's distance (Ling and Okada, $2007)$ in their calculations. $M M_{P D}$, on the other hand, is 44 times faster than both algorithms by use of the sieve approach in the layered matching and the performance gain due to primal dual method.
Table 1. Success rate and average running time for various algorithms on COIL-20 dataset experiment.

\begin{tabular}{|c|c|c|}
\hline Method & $\begin{array}{c}\text { Success Rate } \\
(\mathrm{kNN}=1)\end{array}$ & $\begin{array}{c}\text { Average Time } \\
(\mathrm{sec})\end{array}$ \\
\hline \hline$M M_{L P-\text { Layer } 1}$ & 30.07 & 0.004 \\
$M M_{L P-\text { Layer } 2}$ & 51.6 & 0.008 \\
$M M_{L P-\text { Layer } 3}$ & 77.99 & 0.304 \\
$M M_{L P-\text { Layer } 4}$ & 89.24 & 2.216 \\
$M M_{L P-\text { Layer } 5}$ & 91.11 & 5.943 \\
\hline$M M_{L P-\text { Sieve }}$ & 90 & 0.650 \\
\hline$M M_{P D-\text { Sieve }}$ & 87.64 & 0.033 \\
\hline \hline$M L_{Q P}$ & 92.01 & 2.132 \\
\hline$M L_{L P}$ & 92.08 & 16.761 \\
\hline
\end{tabular}

\section{Conclusion and Future Work}

In this paper, we have presented a novel many-to-many matching method for metric distributions in general and graphs in particular. Our method utilizes the spatial distribution of nodes as well as the node features for the matching process. We use the HST representations of metric distributions to achieve many-to-many matching where the internal nodes of the HSTs capture the segmentation of features. Utilizing hierarchical structure of HSTs, our method allows to do matching at different levels of detail. We also provided an efficient primal-dual approximation algorithm for our method which improves the performance 500 orders of manitude compared to baseline algorithm and 44 times relative to comparable graph based methods in the literature. In a series of experiments in the domain of image and shape matching, we demonstrated that our method performs well both in terms of accuracy and running time.

Although we applied our method to only image matching problem, our technique is general and can be applied to any domain that defines a metric. Performance of the method can further be improved by introducing a distributed version of the primal-dual algorithm. Another direction for future study is to extend the formulation in a way that some of the labels can be discarded from matching calculations.

\section{Acknowledgments}

This work is supported in part by support of the National Science Foundation under Grant Number CNS-1027965.

\section{References}

Almohamad, H., Duffuaa, S.O., 1993. A linear programming approach for the weighted graph matching problem. Pattern Analysis and Machine Intelligence, IEEE Transactions on 15, 522-525.

Ambauen, R., Fischer, S., Bunke, H., 2003. Graph edit distance with node splitting and merging, and its application to diatom identification, in: Graph Based Representations in Pattern Recognition. Springer, pp. 95-106.

Bartal, Y., 1996. Probabilistic approximation of metric spaces and its algorithmic applications, in: Proceedings of the $37^{\text {th }}$ Annual Symposium on Foundations of Computer Science, IEEE Computer Society, Washington, DC, USA. pp. 184-. URL: http://dl.acm.org/citation.cfm?id= 874062.875536

Berretti, S., Del Bimbo, A., Pala, P., 2004. A graph edit distance based on node merging, in: Image and Video Retrieval. Springer, pp. 464-472. 
Bunke, H., 1999. Error correcting graph matching: On the influence of the underlying cost function. Pattern Analysis and Machine Intelligence, IEEE Transactions on 21, 917-922.

Caelli, T., Kosinov, S., 2004. An eigenspace projection clustering method for inexact graph matching. Pattern Analysis and Machine Intelligence, IEEE Transactions on 26, 515-519.

Cook, S.A., 1971. The complexity of theorem-proving procedures, in: Proceedings of the third annual ACM symposium on Theory of computing, ACM. pp. 151-158.

Dantzig, G.B., Ford Jr, L.R., Fulkerson, D.R., 1956. A primal-dual algorithm. Technical Report. DTIC Document.

Demirci, M.F., Osmanlioglu, Y., Shokoufandeh, A., Dickinson, S., 2011. Efficient many-to-many feature matching under the 11 norm. Computer Vision and Image Understanding 115, 976-983.

Demirci, M.F., Shokoufandeh, A., Keselman, Y., Bretzner, L., Dickinson, S., 2006. Object recognition as many-to-many feature matching. International Journal of Computer Vision 69, 203-222.

Fakcharoenphol, J., Rao, S., Talwar, K., 2003. A tight bound on approximating arbitrary metrics by tree metrics, in: Proceedings of the $35^{\text {th }}$ Annual ACM Symposium on Theory of Computing, ACM, New York, NY, USA. pp. 448455. URL: http://doi.acm.org/10.1145/780542.780608, doi:10. 1145/780542.780608.

Goemans, M.X., Williamson, D.P., 1997. The primal-dual method for approximation algorithms and its application to network design problems, in: Hochbaum, D.S. (Ed.), Approximation Algorithms for NP-hard Problems. PWS Publishing Co., Boston, MA, USA, pp. 144-191. URL: http: //dl.acm.org/citation. cfm?id=241938. 241942.

Haxhimusa, Y., Kropatsch, W.G., Pizlo, Z., Ion, A., 2009. Approximative graph pyramid solution of the e-tsp. Image and Vision Computing 27, 887-896.

Huttenlocher, D.P., Klanderman, G.A., Rucklidge, W.J., 1993. Comparing images using the hausdorff distance. Pattern Analysis and Machine Intelligence, IEEE Transactions on 15, 850-863.

Indyk, P., 2001. Algorithmic applications of low-distortion geometric embeddings, in: Proceedings of the $42^{\text {nd }}$ IEEE Symposium on Foundations of Computer Science, IEEE Computer Society, Washington, DC, USA. pp. 10. URL: http://dl . acm.org/citation. cfm?id=874063.875596.

Karp, R.M., 1989. A 2k-competitive algorithm for the circle. Manuscript, August 5.

Keselman, Y., Shokoufandeh, A., Demirci, M.F., Dickinson, S., 2003. Manyto-many graph matching via metric embedding, in: Computer Vision and Pattern Recognition, 2003. Proceedings. 2003 IEEE Computer Society Conference on, IEEE. pp. I-850.

Kleinberg, J., Tardos, E., 2002. Approximation algorithms for classification problems with pairwise relationships: Metric labeling and markov random fields. J. ACM 49, 616-639. URL: http://doi.acm.org/10.1145/ 585265.585268, doi:10.1145/585265.585268.

Ling, H., Okada, K., 2007. An efficient earth mover's distance algorithm for robust histogram comparison. Pattern Analysis and Machine Intelligence, IEEE Transactions on 29, 840-853.

Lowe, D.G., 2004. Distinctive image features from scale-invariant keypoints. International journal of computer vision 60, 91-110.

Nene, S.A., Nayar, S.K., Murase, H., et al., 1996. Columbia object image library (COIL-20). Technical Report. Technical Report CUCS-005-96.

Osmanlıoğlu, Y., Shokoufandeh, A., 2015. Multi-layer tree matching using hsts, in: Graph-Based Representations in Pattern Recognition. Springer International Publishing, pp. 198-207.

Shapiro, L.G., Haralick, R.M., 1981. Structural descriptions and inexact matching. Pattern Analysis and Machine Intelligence, IEEE Transactions on , 504-519.

Wildman, J., Osmanlioglu, Y., Weber, S., Shokoufandeh, A., 2015a. Delay minimizing user association in cellular networks via hierarchically wellseparated trees. arXiv preprint arXiv:1501.02419.

Wildman, J., Osmanlioglu, Y., Weber, S., Shokoufandeh, A., 2015b. Delay minimizing user association in cellular networks via hierarchically wellseparated trees. arXiv preprint arXiv:1501.02419.

Williams, M.L., Wilson, R.C., Hancock, E.R., 1999. Deterministic search for relational graph matching. Pattern Recognition 32, 1255-1271.

Zaslavskiy, M., Bach, F., Vert, J.P., 2010. Many-to-many graph matching: a continuous relaxation approach, in: Machine Learning and Knowledge Discovery in Databases. Springer, pp. 515-530. 


\section{Graphical Abstract (Optional)}

To create your abstract, please type over the instructions in the template box below. Fonts or abstract dimensions should not be changed or altered.

\section{Multilayer Matching of Metric Structures Using \\ Hierarchically Well-separated Trees \\ Yusuf Osmanlıoğlu and Ali Shokoufandeh}

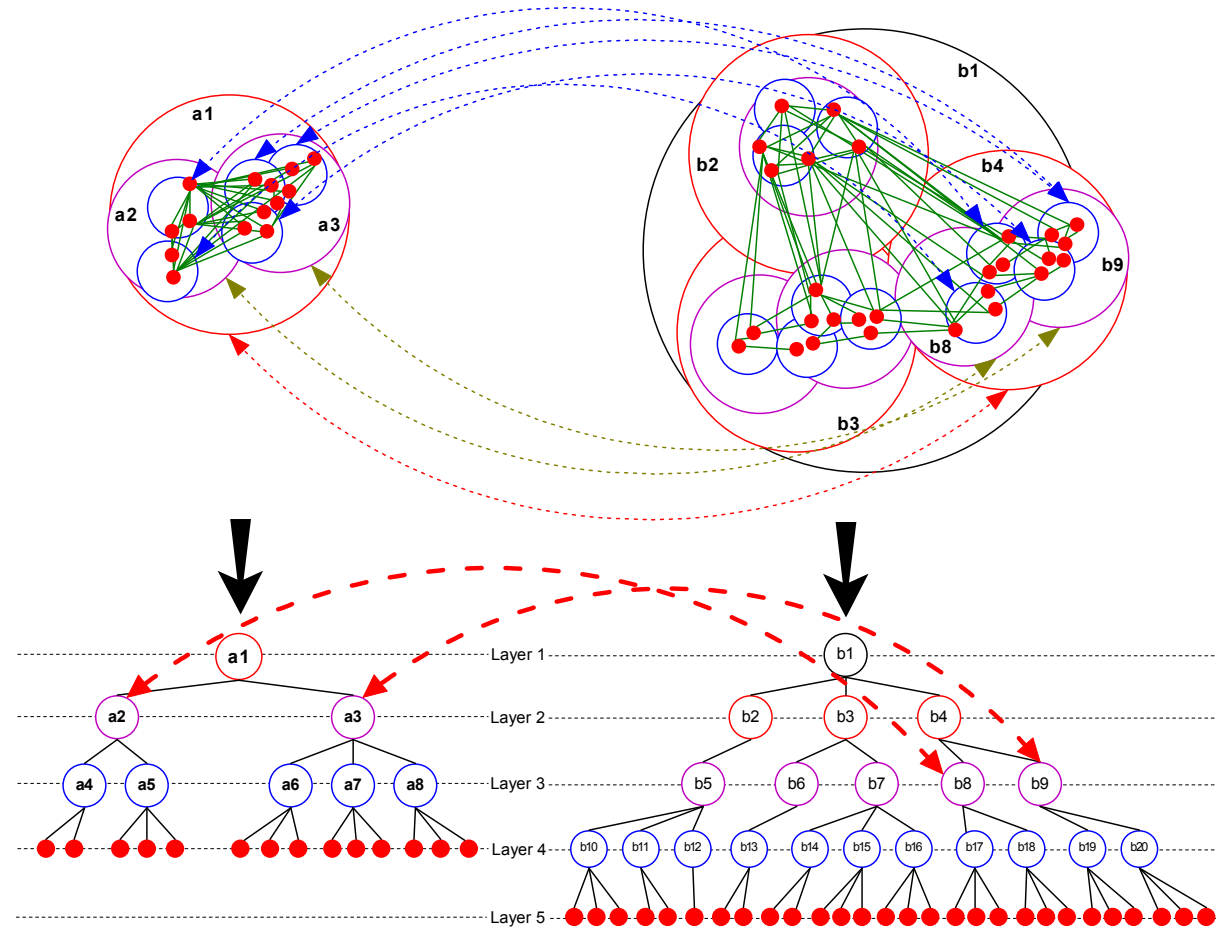

